RESCEU-25/10

\title{
Auxiliary fields representation for modified gravity models
}

\author{
Davi C. Rodrigues* \\ Departamento de Física, CCE, Universidade Federal do Espírito Santo, 29075-910, Vitória, ES, Brazil \\ Filipe de O. Sallest and Ilya L. Shapirdt \\ Departamento de Física, ICE, Universidade Federal de Juiz de Fora, 36036-330, MG, Brazil \\ Alexei A. Starobinsky \\ Landau Institute for Theoretical Physics, Moscow, 119334, Russia \\ RESCEU, Graduate School of Science, The University of Tokyo, Tokyo 113-0033, Japan
}

We consider tensor-multiscalar representations for several types of modified gravity actions. The first example is the theory with the action representing an arbitrary smooth function of the scalar curvature $R$ and $\square R$, the integrand of the Gauss-Bonnet term and the square of the Weyl tensor. We present a simple procedure leading to an equivalent theory of a space-time metric and four auxiliary scalars and specially discuss calibration of a cosmological constant and the condition of the existence of $d S$-like solutions in the case of empty universe. The condition for obtaining a smaller number of independent scalar fields is derived. The second example is the Eddington-like gravity action. In this case we show, in particular, the equivalence of the theory to GR with the cosmological constant term, with or without use of the first-order formalism, and also discuss some possible generalizations.

PACS numbers:

\section{INTRODUCTION}

Recently, there was a considerable interest in the $f(R)$ theories described by the action

$$
S_{f}=\int d^{4} x \sqrt{-g} f(R)
$$

\footnotetext{
*Electronic address: davirodrigues.ufes@gmail.com

${ }^{\dagger}$ Electronic address: salles@ice.ufjf.br

${ }^{\ddagger}$ Electronic address: shapiro@fisica.ufjf.br . Also at Tomsk State Pedagogical University, Tomsk, Russia.

${ }^{\S}$ Electronic address: alstar@landau.ac.ru
} 
where $f(R)$ is some differentiable function, see [1] for recent reviews. It is well-known that, under the condition $f^{\prime \prime}(R) \neq 0$, the theory is dynamically equivalent to scalar-tensor theory of gravity with the potential depending on the form of the function $f(R)$. Our purpose is to discuss this equivalence in a slightly different framework. As an application of our method we will be able to generalize the equivalence theorem to the more general case when the action depends on a function of many variables, $f\left(X^{i}\right)$, with $X^{i}$ being, e.g., $R, \square R$, Gauss-Bonnet integrand $E$ and/or other quantities. Let us note that quantum corrections to GR (coming from the semiclassical approach to quantum gravity or from the string theory) can be modelled by such a function to some extent. Another advantage of the method which we present here is that it can be used, also, for other theories, e.g. for Eddington-like models. Last, but not least, our method is a bit more explicit and simple than the previously known ones, e.g., [2 8] $)$. All considerations will be presented for the $D=4$ case in order to make them more explicit, but they can be more or less straightforwardly generalized for an arbitrary $D \neq 2$ case.

In the present paper we will mainly follow Lagrangian approach, but it is worthwhile to mention that there are also some recent papers treating $f(R)[9]$ and even more general $f\left(R_{\mu \nu \alpha \beta}\right)$ [10] theories within the canonical formalism. Earlier, the discussion of stability issues for a while class of higher derivative theories has been presented in [11] at both classical level and also for the Wheeler-DeWitt equation. In general, the equivalence of two theories at the classical level does not imply their equivalence at the quantum level. The quantum treatment of higher derivative and scalar-tensor theories is not a trivial issue, hence here we present a short discussion of it only.

The paper is organized as follows. In Sect. 2 we consider the simplest case of the theory (11) and describe a simple way of mapping it into a metric-scalar (scalar-tensor) model. The content of this section is mainly not original, we just give a bit more simple form of the known transformations. One of relatively new aspects of our consideration is the procedure to fix the cosmological constant term in the metric-scalar representation of the theory. We also check this procedure by using the dS-like exponential solution. In Sect. 3 we address gravity theories of a rather generic form, in which the action contains an arbitrary function of various scalar, curvature-dependent invariants, such as the scalar curvature $R$, the Gauss-Bonnet term, the square of the Weyl tensor and others. We develop a systematic approach to map such theories into the metric-multiscalar models. One of the new elements of our consideration is that it includes the case when the initial theory has such Hamiltonian constraints that the number of independent auxiliary scalars is smaller than the number of initial curvature-dependent invariants. The condition for this to occur is obtained. Sect. 4 is devoted to the formulation of general conditions for the existence of exponential solutions in 
the theories investigated in Sect. 3. In Sect. 5 we consider the theory which is based on the string low-energy effective action of gravity, up to the third order in curvature invariants. In this case one cannot construct an equivalent scalar-tensor representation at the level of action or general equations of motion for the metric field. However, this problem can be perfectly addressed for the much more restricted case of a homogeneous and isotropic cosmological solution. In Sect. 6 we apply our method of constructing equivalent theories to the wide class of the Eddington-like gravity theories. It is shown how to construct dual theories for such gravity theories, including both the second order and Palatini formalisms. In the latter case the auxiliary tensor field can be interpreted as a space-time metric. In Sect. 7 we discuss the equivalence between different representations of the theory (11) and its generalizations at the quantum level. Finally, in Sect. 8 we draw our conclusions.

\section{EQUIVALENCE OF $f(R)$ AND METRIC-SCALAR THEORY}

Let us start from a simple pedagogical example of the theory (10) and find its metric-scalar (scalar-tensor) dual. Consider the theory described by the action

$$
S_{1}=\int d^{4} x \sqrt{-g}\{\psi R-V(\psi)\}
$$

The theory (2) describes a dynamical scalar $\psi$, despite there is no kinetic term for $\psi$ in the action. One can establish the relation between the theories (1) and (2). The equation of motion which follows from the variation of $\psi$ in (2) has the form

$$
R=V^{\prime}(\psi)=\frac{d V}{d \psi} .
$$

After solving (3) with respect to $\psi$ and substituting this solution $\psi=\psi(R)$ back into (2), we obtain the action (11) with

$$
\psi(R) \cdot R-V(\psi(R))=f(R)
$$

This means that the equivalence of the two actions is dynamical, i.e. it holds on extremal curves of the field $\psi$. Later on we shall confirm the validity of this procedure through the equations of motion for both metric and $\psi$, i.e. in a way similar to the one of [5].

Our next step will be to find the relation between the functions $V(\psi)$ and $f(R)$. Taking the derivative $d / d R$ of Eq. (44), we arrive at the relation

$$
\psi+R \psi^{\prime}(R)-V^{\prime}(\psi) \psi^{\prime}(R)=f^{\prime}(R)
$$


In this formula we assume that $\psi=\psi(R)$ and $R=R(\psi)$. Using (3), the equation (5) immediately reduces to the very simple relation

$$
\psi=f^{\prime}(R)
$$

indicating that the function $R=V^{\prime}(\psi)$ is nothing else but the inverse to the function $\psi=f^{\prime}(R)$.

Finally, we arrive at the following receipt for deriving the potential $V(\psi)$ for a given $f(R)$.

a) Calculate $\psi=f^{\prime}(R)$ and invert it, obtaining $R=V^{\prime}(\psi)$. Note that the possibility of such inversion requires $f^{\prime \prime}(R) \neq 0$.

b) Integrate over $\psi$ :

$$
V(\psi)=\Omega_{0}+\int_{0}^{\psi} R(\psi) d \psi
$$

One has to note that an arbitrary integration constant $\Omega_{0}$ in (17) exactly corresponds to the constant $f_{0}=f(R=0)$ component of the integrand of Eq. (1), which is indeed lost when we take the derivative $f^{\prime}(R)$. Furthermore, in order to fix the constant $\Omega_{0}$, one can use the following simple consideration. By using (4) we arrive at

$$
V(\psi)=R \psi-f(R), \quad \text { where } \quad \psi=f^{\prime}(R) .
$$

Remember that when placed into the covariant action, $\Omega_{0}$ can not be regarded as an irrelevant constant, because it is multiplied by the metric-dependent factor $\sqrt{-g}$. As far as (77) should be equal to (8), one can then fix $\Omega_{0}$. Later one we will additionally check the validity of this procedure for a cosmological $d S$-like solution.

The prescription given above enables one, in principle, to find the potential function $V(\psi)$ for a given $f(R)$. Let us check the results of this simple procedure at the level of the equations of motion. Taking variation of the equation (1) with respect to the metric, we obtain

$$
f^{\prime}\left(R_{\mu \nu}-\frac{1}{2} R g_{\mu \nu}\right)+\frac{1}{2} g_{\mu \nu}\left(R f^{\prime}-f\right)-\nabla_{\mu} \nabla_{\nu} f^{\prime}+g_{\mu \nu} \square f=0 .
$$

Performing the same operation for (2), we arrive at

$$
\psi\left(R_{\mu \nu}-\frac{1}{2} R g_{\mu \nu}\right)=-\frac{1}{2} g_{\mu \nu} V(\psi)+\nabla_{\mu} \nabla_{\nu} \psi-g^{\mu \nu} \square \psi=0 .
$$

One can verify that the equivalence between (9) and (10) holds if the relation

$$
\frac{f^{\prime} R-f}{f^{\prime}}=\frac{V(\psi)}{\psi}
$$

is satisfied. It is easy to check that the solution of this equation has the form (6). 
Consider some particular example for the procedure described above. The simplest case leading to the linear equations $\psi=f^{\prime}(R)$ is

$$
f(R)=\Omega-\kappa^{2} R+\frac{\alpha}{2} R^{2}
$$

Using our previous results, one can easily arrive at

$$
\psi(R)=f^{\prime}(R)=-\kappa^{2}+\alpha R \quad \Longrightarrow \quad V^{\prime}(\psi)=R=\frac{\psi+\kappa^{2}}{\alpha} .
$$

Integrating (13) we get

$$
V(\psi)=\Omega_{0}+\frac{\psi^{2}}{2 \alpha}+\frac{\kappa^{2} \psi}{\alpha}
$$

Finally, in order to fix the integration constant, one has to put $\psi=-\kappa^{2}+\alpha R$ back into (14) and compare it to (12). This procedure gives us

$$
\Omega=\Omega_{0}-\frac{\kappa^{4}}{2}
$$

One can perform a simple verification of the described procedure for fixing $\Omega$. For this end, we will now derive the $d S$-like solution for both theories (11) and (21) in case of (12). The metric of our interest is

$$
d s^{2}=g_{\mu \nu} d x^{\mu} d x^{\nu}=d t^{2}-a^{2}(t)\left(\frac{1}{1-k r^{2}}+r^{2} d \Omega\right)
$$

$a(t)=\exp \{\sigma(t)\}$ and afterwards we will set $\sigma(t)=H_{0} t$. It is easy to obtain the equation for $\sigma$ for the theory (2),

$$
\frac{1}{\sqrt{-g}} \frac{\delta S_{1}}{\delta \sigma}=-6 e^{-2 \sigma}\left(2 \psi k+\psi^{\prime \prime}+2 \sigma^{\prime \prime} \psi+2 \psi^{\prime} \sigma^{\prime}+2 \sigma^{2} \psi\right)-4 V(\psi)=0 .
$$

Here the prime stands for the derivative with respect to the conformal time, e.g.,

$$
\sigma^{\prime}=\frac{d \sigma}{d \eta}=a(t) \frac{d \sigma}{d t}
$$

while the derivative with respect to the physical time $t$ is denoted as a dot. It terms of the physical time and adopting $k=0$, we obtain the relation

$$
-12 H_{0}^{2} \psi-9 H_{0} \dot{\psi}-3 \ddot{\psi}=2 \mathrm{~V}
$$

Using $\psi=-\kappa^{2}+\alpha R$ and taking into account $R=-12 H_{0}^{2}$ for the FRW metric, we get

$$
V=6 H_{0}^{2} \kappa^{2}-6 H_{0}^{2} \alpha R
$$


and finally

$$
\Omega=6 H_{0}^{2} \kappa^{2}
$$

On the other hand, starting from

$$
S_{f}=\int d^{4} x \sqrt{-g} f(R)=\int d^{4} x \sqrt{-g}\left[\Omega-\kappa^{2} R+\frac{\alpha}{2} R^{2}\right]
$$

we arrive at the equation

$$
\frac{1}{\sqrt{-g}} \frac{\delta S_{f}}{\delta \sigma}=4 \Omega-6 \kappa^{2} e^{-2 \sigma}\left(2 \sigma^{\prime 2}+2 \sigma^{\prime \prime}\right)+18 \alpha e^{-4 \sigma}\left(2 \sigma^{\prime \prime \prime \prime}-12 \sigma^{\prime 2} \sigma^{\prime \prime}\right)=0 .
$$

It is straightforward to check that the solution $\sigma=H_{0} t=-\ln \left(H_{0}|\eta|\right)$ corresponds, again, to the relation (19).

Finally, for the sake of completeness, let us address the possibility of using conformal transformation to deal with the metric-scalar theory. It is well known fact that the theory (2) can be easily mapped into another one with the standard form of the scalar kinetic term. We will give the corresponding treatment here just for completeness and refer the reader to the review [12] for further details and (numerous, indeed) references.

Let us start from the conformal transformation

$$
g_{\mu \nu} \longrightarrow \bar{g}_{\mu \nu}=g_{\mu \nu} \cdot e^{2 \sigma(x)}
$$

in the action (2). Simple calculation yields the following result

$$
S_{c}\left[g_{\mu \nu} e^{2 \sigma}, \psi\right]=\int d^{4} x \sqrt{-g}\left\{\psi e^{2 \sigma}\left[R-6(\nabla \sigma)^{2}-6 \square \sigma\right]-e^{4 \sigma} V(\psi)\right\},
$$

where $(\nabla \sigma)^{2}=g^{\mu \nu} \partial_{\mu} \sigma \partial_{\nu} \sigma$. Let us choose $\sigma$ such that $\psi e^{2 \sigma}=-\kappa^{2}$. Then the first term $\psi e^{2 \sigma} R$ coincides with the Einstein-Hilbert term, also the third term $-6 \psi e^{2 \sigma} \square \sigma$ becomes a total derivative which does not affect the equations of motion. In order to provide the standard form of the kinetic term, we take

$$
\varphi=2 \sqrt{3} \kappa \sigma, \quad \text { then } \quad \psi=-\kappa^{2} \exp \left\{-\frac{\varphi}{\sqrt{3} \kappa}\right\} .
$$

The output looks like

$$
S_{\min }\left[g_{\mu \nu}, \varphi\right]=\int d^{4} x \sqrt{-g}\left\{-\kappa^{2} R+\frac{1}{2} g^{\mu \nu} \partial_{\mu} \varphi \partial_{\nu} \varphi-U(\varphi)\right\}
$$

where the two potentials are related as

$$
U(\varphi)=e^{4 \sigma} V(\psi)=\frac{\kappa^{4}}{\psi^{2}} V(\psi) .
$$


The formulas (22), (24) and (26) are nothing but the change of variables in the action (2) which transform it into the action (25). Therefore we do not need to check the equivalence between two actions by other means, e.g. examining the equations of motion. Finally, let us note that the multiscalar case can be, in principle, also treated by the conformal transformation, but this transformation is not so easy as in the one-scalar case [13].

\section{MODIFIED GRAVITY THEORY OF A MORE GENERAL FORM}

We have presented a useful and simple prescription of mapping theories (11) into theories (2) at the classical level. This method can be generalized to the gravitational actions which are more general than (11). However, as we will see in what follows, in this case one needs more scalar fields. Some similar results has been recently obtained in [14] and [15], but the derivation there looks rather different.

The method may be especially useful for working out the dS-type solutions and, therefore, is applicable for testing various models of modified gravity, including the ones corresponding to quantum corrections. Consider the following gravitational action

$$
S_{g e n}=\int d^{4} x \sqrt{-g} f\left(R, \square R, C^{2}, \widetilde{E}\right),
$$

where $\widetilde{E}=E-\frac{2}{3} \square R, \quad E=R_{\mu \nu \alpha \beta}^{2}-4 R_{\alpha \beta}^{2}+R^{2}$ is the Gauss-Bonnet topological term (Euler density) and $C^{2}=R_{\mu \nu \alpha \beta}^{2}-2 R_{\alpha \beta}^{2}+(1 / 3) R^{2}$ is the square of the Weyl tensor. In view of the cosmological applications, it proves more useful to consider $\widetilde{E}$ rather than $E$.

We start by introducing a generalization of the action (11),

$$
S_{1}=\int d^{4} x \sqrt{-g} f\left(X_{i}\right) \quad \text { where } \quad X_{i}=R, \square R, \widetilde{E}, C^{2}
$$

in case of the action (27), but the number of invariants can be easily extended. For this end we set $i=1, \ldots, N$. Consider the dual action

$$
S_{2}=\int d^{4} x \sqrt{-g}\left[X_{i} \psi^{i}-V\left(\psi^{i}\right)\right]
$$

where repeated indices imply summation, as usual. Let us follow the same scheme which we applied in the previous section. The equations for the $\psi^{i}$ have the form

$$
X_{i}=\frac{\partial V}{\partial \psi^{i}} .
$$

We put them into (29), demanding equivalence to the action (28),

$$
S_{2}=\int d^{4} x \sqrt{-g}\left\{\psi^{i} \frac{\partial V}{\partial \psi^{i}}-V\left(\psi^{i}\right)\right\}=\int d^{4} x \sqrt{-g} f\left(X_{i}\right) .
$$


Assuming

$$
f\left(X_{i}\right)=X_{i} \psi^{i}-V\left(\psi^{i}\right)
$$

and taking partial derivatives with respect to $X^{i}$ in (32), we arrive at

$$
\frac{\partial f}{\partial X_{k}}=\psi^{k}+X_{i} \frac{\partial \psi^{i}}{\partial X_{i}}-\frac{\partial V}{\partial \psi^{i}} \frac{\partial \psi^{i}}{\partial X_{k}} \Longrightarrow \psi^{k}=\frac{\partial f}{\partial X_{k}},
$$

where we used Eq. (30). The formulas (30) and (33) show that we always have

$$
X_{i}=\frac{\partial V(\psi)}{\partial \psi_{i}} \quad \text { and } \quad \psi_{k}=\frac{\partial f(X)}{\partial X_{k}}
$$

After all, the prescription for deriving $V\left(\psi^{i}\right)$ is very similar to the one described in the previous section and looks as follows:

a) calculate $\psi^{k}=\frac{\partial f(X)}{\partial X_{k}}$;

b) solve these equations and find $X_{k}(\psi)=\frac{\partial V}{\partial \psi^{k}}$;

c) integrate the last relations and find $V\left(\psi_{k}\right)$ up to the additive constant;

d) fix this constant by the requirement that the actions coincide in the corresponding limit (typically zero curvature).

One can note that this procedure can be applied also to the non-Riemannian generalizations of GR, including the theory of gravity with torsion.

Let us consider an example of how the equivalent metric-scalar theory can be achieved. We start from the action (27) with the function

$$
f(R, \widetilde{E})=F(R) \cdot \widetilde{E}
$$

where $F(R)$ is an arbitrary function of scalar curvature, which will be fixed later on. The equivalent action is

$$
S_{2}=\int d^{4} x \sqrt{-g}\{\psi R+\chi \widetilde{E}-V(\psi, \chi)\}
$$

Let us follow the prescription described above. The equations

$$
\frac{\partial f}{\partial \widetilde{E}}=\chi=F(R), \quad \frac{\partial f}{\partial R}=\psi=\widetilde{E} F^{\prime}(R)
$$

can be solved with respect to the two scalar fields,

$$
R=g(\chi), \quad \widetilde{E}=\frac{\psi}{F^{\prime}(R)} \quad \Longrightarrow \quad \widetilde{E}=\frac{\psi}{F_{g}^{\prime}(g(\chi))} .
$$


On the other hand, we have inverse functions

$$
R=\frac{\partial V}{\partial \psi}=g(\chi), \quad \widetilde{E}=\frac{\partial V}{\partial \chi}=\frac{\psi}{F_{g}^{\prime}(g(\chi))}
$$

Then

$$
V(\psi, \chi)=\int g(\chi) d \psi+g_{1}(\chi)=g_{1}(\chi)+\psi g(\chi)
$$

and

$$
V(\psi, \chi)=\int d \chi \frac{\psi}{F_{g}^{\prime}(g(\chi))}+g_{2}(\psi)=\psi \int \frac{d \chi}{F_{g}^{\prime}(g(\chi))}+g_{2}(\psi)
$$

If we compare the two forms of the potential function (40) and (41), it becomes clear that $g(\chi)$ satisfies the functional equation

$$
g(\chi)=\int \frac{d \chi}{F_{g}^{\prime}(g(\chi))}+C
$$

and, moreover,

$$
g_{2}(\psi)-C \psi=g_{1}(\chi)=C_{1}=\text { const } .
$$

It does not look possible to advance further, so let us take a more concrete form of $F(R)$. Consider first a very simple case $f(R, \widetilde{E})=-\Omega+R \widetilde{E}$. Making the same steps as in the general case, we obtain

$$
F(R)=R \Longrightarrow \chi=R, \quad g(\chi)=\chi, \quad F^{\prime}(R)=1 .
$$

Using (40) and (41), one obtain

$$
V(\psi, \chi)=g_{1}(\chi)+\psi \chi=\psi \int d \chi+g_{2}(\psi)=\psi \chi+g_{2}(\psi) .
$$

It is easy to see that in this case $g_{1}(\chi)=g_{2}(\psi)=C$, so we get

$$
V(\psi, \chi)=\psi \chi+C
$$

Finally, inserting the relations $\chi=R$ and $\psi=\widetilde{E}$ into (44), one can easily verify that $C=\Omega$. This result can be also checked by inspecting exponential solutions in the two cases. We avoid to bother the reader with the details of this verification, but just note that its output is positive.

Consider a bit more complicated example when

$$
f(R, \widetilde{E})=\Omega-\kappa^{2} R+\beta \widetilde{E} \ln \left(1+\frac{R}{R_{0}}\right),
$$


where $\beta$ is some constant and $R_{0}$ is a reference value for the scalar curvature. The expression (45) can be seen as a part of the renormalization group corrected vacuum action, where the renormalization group parameter $\mu^{2}$ is associated to the scalar curvature (see, e.g., [16] for further details and references).

Following the footsteps of the previous examples, we derive

$$
\frac{\partial f(R, \widetilde{E})}{\partial \widetilde{E}}=\chi=\beta \ln \left(1+\frac{R}{R_{0}}\right) \quad \Longrightarrow \quad R=R_{0}\left(e^{\chi / \beta}-1\right)
$$

and

$$
\frac{\partial f(R, \widetilde{E})}{\partial R}=\psi=\frac{\beta \widetilde{E}}{R+R_{0}}-\kappa^{2} \Longrightarrow \widetilde{E}=\frac{R_{0}}{\beta}\left(\psi+\kappa^{2}\right) e^{\chi / \beta}
$$

At the next stage we find

$$
\begin{aligned}
& \frac{\partial V(\psi, \chi)}{\partial \psi}=R \quad \Longrightarrow \quad V(\psi, \chi)=g_{1}(\chi)+R_{0} \psi e^{\chi / \beta}-R_{0} \psi . \\
& \frac{\partial V(\psi, \chi)}{\partial \chi}=\widetilde{E} \quad \Longrightarrow \quad V(\psi, \chi)=g_{2}(\psi)+R_{0} \psi e^{\chi / \beta}+\kappa^{2} R_{0} e^{\chi / \beta} .
\end{aligned}
$$

Using these two expressions it is easy to figure out that

$$
g_{1}(\chi)=\kappa^{2} R_{0} e^{\chi / \beta}+C \text { and } g_{2}(\psi)=-R_{0} \psi+C
$$

Finally, we arrive at the potential

$$
V(\psi, \chi)=R_{0} \psi\left(e^{\chi / \beta}-1\right)+\kappa^{2} R_{0} e^{\chi / \beta}-\Omega-\kappa^{2} R_{0},
$$

where the constant $C=-\Omega-\kappa^{2} R_{0}$ has been fixed following the same method which we used in the previous cases.

One can consider more complicated expression for the covariant Lagrangian,

$$
f\left(R, \widetilde{E}, C^{2}\right)=\Omega-\kappa^{2} R+\beta \widetilde{E} \ln \left(1+\frac{R}{R_{0}}\right)+\beta_{1} C^{2} \ln \left(1+\frac{R}{R_{0}}\right) .
$$

At this point one can make an important observation. From the first sight, the equivalent Lagrangian for this case should have three auxiliary fields, because there are three structures $R, \widetilde{E}$ and $C^{2}$. At the same time, the problem of reducing the theory (49) is essentially equivalent to the one of the theory (45), with the $\beta \widetilde{E}$ traded by the combination $\beta \widetilde{E}+\beta_{1} C^{2}$. Obviously, in this case we need only two auxiliary fields and not three of them. In other words, in this case the number of necessary auxiliary fields is smaller than the one which could be thought by just counting the number of the structures $X_{i}$ in the starting action. This example shows that it would be interesting 
to have a general criteria for establishing an exact number of necessary auxiliary fields for a given initial function $f\left(X_{i}\right)$.

The problem of our interest is very close to the one which is typical for the transition from Lagrange to Hamiltonian formalism in the theory with constraints [47] (see, e.g., well-known books [18, 19] for introduction purposes). Indeed, it is analogous to the passage from the Lagrangian description, with no explicit dependence on the coordinates, to the Hamiltonian one. In this case $f\left(X^{i}\right)$ and $X^{i} \psi_{i}-V\left(\psi_{i}\right)$ play the roles of the Lagrangian and the Hamiltonian respectively, where the "momenta" are defined by

$$
\psi_{i} \equiv \frac{\partial f}{\partial X^{i}}
$$

Finally, the quantities of $\left\{X^{1}, X^{2}, X^{3}\right\}$ play the roles of "velocities". For the specific case of eq. (49), these equations have the form

$$
\begin{aligned}
& \psi_{1}=-\kappa^{2}+\frac{\beta \tilde{E}+\beta_{1} C^{2}}{R_{0}+R}, \\
& \psi_{2}=\beta \ln \left(1+\frac{R}{R_{0}}\right), \\
& \psi_{3}=\beta_{1} \ln \left(1+\frac{R}{R_{0}}\right),
\end{aligned}
$$

from what we directly infer the presence of a constraint, which is a dependence relation between the $\psi_{i}$ given by

$$
\phi \equiv \beta_{1} \psi_{2}-\beta \psi_{3} \equiv \beta_{1} \chi-\beta \chi_{1}=0
$$

Since the constraint $\phi\left(\psi_{i}\right)$ comes directly from the definition of the "momenta", it is classified as a primary constraint [18, 19]. We note that the equation $\phi\left(\psi_{i}\right)=0$ defines a surface in the space $\left\{\psi_{1}, \psi_{2}, \psi_{3}\right\}$. It should be stressed that this constraint only represents a restriction in the "momenta" space, while in the "velocity" space $\left\{X^{1}, X^{2}, X^{3}\right\}$ the constraint does not lead to any restrictions, since if the $\psi_{i}$ are written as functions of the $X^{k}$, the constraint $\phi\left(\psi_{i}\right)$ becomes the function $\phi\left(\psi_{i}\left(X^{k}\right)\right)$, which is identically null.

If this were a typical Hamiltonian problem, one would evaluate the evolution of the primary constraints (e.g., the single one in the example considered above) in search for further constraints. However our present problem is simpler, because it does not involve any dynamics. Hence only the constraints with no relation to dynamics are relevant here, which are the primary ones.

Before proceeding towards the determination of the potential $V$, we remark here on the relation 
between the Hessian matrix

$$
\left(\frac{\partial^{2} f}{\partial X^{i} \partial X^{j}}\right)
$$

and the presence of constraints. Firstly, if the Hessian is non-singular, the inverse function theorem guarantees that (at least locally) one can use the definition (50) to express the $X^{k}$ as a function of the $\psi_{i}$, and thus no constraint is expected. However the Hessian can turn out to be degenerate. For instance, in the case of (49), this $3 \times 3$ matrix is a singular matrix of rank two (for $R \neq-R_{0}$ ), namely

$$
\left(\frac{\partial^{2} f}{\partial X^{i} \partial X^{j}}\right)=\frac{1}{\left(R+R_{0}\right)^{2}}\left(\begin{array}{cccc}
-\tilde{E} \beta+C^{2} \beta_{1} & \beta & \beta_{1} \\
\beta & 0 & 0 \\
\beta_{1} & 0 & 0
\end{array}\right)
$$

Therefore, it has a single independent zero-mode (i.e., an eigenvector whose corresponding eigenvalue is zero). Indeed,

$$
\nu=\left(\begin{array}{lll}
0 & \beta_{1} & -\beta
\end{array}\right)
$$

can be promptly identified as the single linearly independent zero-mode of the Hessian (55). Let us note that we choose to work with the zero-modes that multiply the Hessian matrix by the left.

To conclude this introductory part, we note that each independent zero-mode generates an independent constraint. In particular, by multiplying the zero-mode $\nu$ on both sides of the definition (50), one finds the same constraint (54). Afterwards we will show that each independent constraint leads to an independent zero-mode of the Hessian matrix. We note that this simple relation between zero-modes and constraints does not have a counterpart in general Hamiltonian problems with constraints, in particular since the corresponding zero-modes may depend on "coordinates" there (in the present problem, we are considering the analogous Hamiltonian problem in which the Lagrangian only depend on the "velocities" $X^{i}$ ).

In the presence of constraints, one cannot use the first relation of (34) to find $V$, since this relation is not valid in the presence of constraints. Namely, consider the variation of $V$ in the constraint surface (i.e., in the surface $\phi=0$ ),

$$
\delta V=\delta\left(\psi_{i} X^{i}-f\right)=X^{i} \delta \psi_{i}+\left(\psi_{i}-\frac{\partial f}{\partial X^{i}}\right) \delta X^{i}=X^{i} \delta \psi_{i}
$$

In the last step above, we used the definition of the momenta (50). The previous equation shows that $V$ can be written as a function of $\psi$ alone, even if constraints are present. Thus, using the 
last equality,

$$
\left(\frac{\partial V}{\partial \psi_{i}}-X^{i}\right) \delta \psi_{i}=0
$$

Now, using the Theorem 1.2 of [19], we find the extension of the first relation of (34) to the constrained case,

$$
X^{i}=\frac{\partial V}{\partial \psi_{i}}+\lambda_{m} \frac{\partial \phi_{m}}{\partial \psi_{i}}
$$

In this formula $\lambda_{m}$ are Lagrange multipliers and $\phi_{m}$, with $m=1,2, \ldots, M$, are all the constraints of the model under consideration. In the particular case of (49) we have a single constraint, i.e. $M=1$. The introduction of these multipliers is necessary since the relation between the $X_{i}$ and the $\psi^{k}$ has to be extended in order to become invertible.

Now we are in a position to discuss the method of constructing potential $V\left(\psi_{i}\right)$ in the case of a theory with constraints. The integration method which was employed previously can be extended to this case. For instance, in the example of $f\left(R, \tilde{E}, C^{2}\right)$ theory (49) one can solve the definition of $\psi_{i}$ and arrive at

$$
\begin{aligned}
& R=R_{0}\left(e^{\chi / \beta}-1\right), \\
& \tilde{E}=\frac{R_{0}}{\beta} e^{\chi / \beta}\left(\psi+\kappa^{2}\right)-\frac{\beta_{1}}{\beta} C^{2} .
\end{aligned}
$$

Hence, from (59) and (54) we find

$$
\begin{aligned}
& R_{0}\left(e^{\chi / \beta}-1\right)=\frac{\partial V}{\partial \psi}, \\
& \frac{R_{0}}{\beta} e^{\chi / \beta}\left(\psi+\kappa^{2}\right)-\frac{\beta_{1}}{\beta} C^{2}=\frac{\partial V}{\partial \chi}+\lambda \beta_{1}, \\
& C^{2}=\frac{\partial V}{\partial \chi_{1}}-\lambda \beta
\end{aligned}
$$

The first equation can be straightforwardly integrated, but the second cannot, since we do not know how to express both $C^{2}$ and $\lambda$ as functions of the $\psi_{i}$. Nevertheless, the Lagrange multiplier is still free, thus we can set it in such a way that the $C^{2}$ term disappear, namely $\lambda=-C^{2} / \beta$. Consequently, the third equation (64) becomes

$$
\frac{\partial V}{\partial \chi_{1}}=0
$$

After that the expression for $V$ can be integrated, and $V\left(\psi_{i}\right)$ can be found using procedures similar to that used in the unconstrained case. One should note that the number of auxiliary fields in this procedure is smaller than the number $N$ of the $X_{i}$ structures in the initial $f\left(X_{i}\right)$ theory. For 
example, in the (49) case, albeit we started from the $f\left(X_{i}\right)$ which depends on three independent quantities, the corresponding potential $V$ only depends on the two independent scalar fields.

The form of the potential $V$ can have some impact on the physical consequences of a given theory $f\left(X_{i}\right)$. Hence, before concluding this example, we present $V$ in a more general form. Let us start, as usual, from the simple example. In Eq. (60) we have made a choice of selecting the particular expression of $R$ as a function of $\chi$ and $\chi_{1}$. At the same time one can express $R$ in a more general form,

$$
R=\frac{R_{0}}{1+\xi}\left[e^{\chi / \beta}-1+\xi\left(e^{\chi_{1} / \beta_{1}}-1\right)\right]
$$

where $\xi$ is an arbitrary real number different from -1 . The choice in (60) corresponds to $\xi=0$. From the more general version (65) and Eq. (51) one can easily obtain

$$
\beta \tilde{E}+\beta_{1} C^{2}=R_{0} \frac{\left(\psi+\kappa^{2}\right)}{\xi+1}\left(e^{\chi / \beta}+\xi e^{\chi_{1} / \beta_{1}}\right) .
$$

Now, instead of attempting a direct integration of $V$ (as in the non-constrained case), we express it on the constraint surface $\phi=0$, as

$$
\begin{aligned}
V=\psi_{i} X^{i}-f & =\psi_{i} X^{i}-\left[\Omega-\kappa^{2} R_{0} \frac{\left(e^{\chi / \beta}-1\right)+\xi\left(e^{\chi_{1} / \beta_{1}}-1\right)}{\xi+1}+\tilde{E} \chi+C^{2} \chi_{1}\right] \\
& =R_{0}\left(\psi+\kappa^{2}\right) \frac{e^{\chi / \beta}-1+\xi\left(e^{\chi 1 / \beta_{1}}-1\right)}{\xi+1}-\Omega \\
& =R_{0}\left(\psi+\kappa^{2}\right)\left(e^{\chi / \beta}-1\right)-\Omega .
\end{aligned}
$$

As one should expect, on the constraint surface $V$ is independent on the value of $\xi$. Nevertheless, a choice of $\xi=1$ or $\xi=0$, for instance, might have computational advantages one over the other. Continuing the integration of $V$ as in the previous method, it is straightforward to check that the final answer is given by (69).

With the experience which we just gained from the example considered above, it is not hard to guess that, in general, there is a straight relation between the number of independent zero-modes of the Hessian and the number of constraints. Consider the case in which there are $M$ independent constraints given by

$$
\phi_{m}\left(\psi_{i}\right)=0
$$

with $m=1,2, \ldots, M$. We assume that the constraints are expressed such that the gradients of the constraints are linearly independent on the constraint surface (for more details, see the regularity conditions on how to express the constraints [19]). 
Since the primary constraints $\phi_{m}(\psi)$ are identically null when expressed as functions of the $X^{i}$ variables,

$$
\frac{\partial \phi_{m}\left(\psi_{k}\left(X^{j}\right)\right)}{\partial X^{i}}=\frac{\partial \phi_{m}\left(\psi_{k}\right)}{\partial \psi_{j}} \frac{\partial^{2} f}{\partial X^{i} \partial X^{j}}=0 .
$$

Then, for each independent constraint $\phi_{m}\left(\psi_{i}\right)$ there is a corresponding independent zero-mode of the Hessian given by

$$
\left(\nu^{j}\right)_{m}=\frac{\partial \phi_{m}}{\partial \psi_{j}}
$$

In other words, upon transforming a Lagrangian given by $f\left(X^{i}\right)$, with $i=1,2, \ldots, N$, into an equivalent one given by $\psi_{i} X^{i}-V\left(\psi_{i}\right)$, the number of independent auxiliary scalar fields that appear in the potential $V\left(\psi_{i}\right)$ is equal to the rank of the Hessian of $f\left(X^{i}\right)$.

In particular, if $f$ depends on $R$ and $E$ only, the condition of the degeneracy of the Hessian matrix reduces to

$$
f_{R R} f_{E E}-f_{R E}^{2}=0
$$

The principal difference between the behaviour of solutions in this special case and in the general one has been already noticed when studying small inhomogeneous perturbations on a FriedmannRobertson-Walker (FRW) background [14].

A relevant general observation is that we only need the potential $V$ computed on the constraint surface, since in general [19]

$$
V=\left.V\right|_{\phi=0}+\lambda \phi
$$

where $\left.V\right|_{\phi=0}$ is the potential on the constraint surface, but the ("primary") constraint $\phi$ identically vanishes when expressed as a function of $R^{\prime} s$. Thus, it does not make any difference whether one uses either $f=X^{i} \psi_{i}-V$ or $f=X^{i} \psi_{i}-\left.V\right|_{\phi=0}$.

\section{EXAMPLE: CONDITIONS FOR EXPONENTIAL INFLATION}

As an illustration of the equivalence theorem from the previous section, let us formulate the conditions for the existence of a strictly exponential cosmological solution. Consider the action

$$
S_{e q}=\int d^{4} x \sqrt{-g}\left\{\psi_{1} R+\psi_{2} \square R+\psi_{3} C^{2}+\psi_{4}\left(E-\frac{2}{3} \square R\right)-V\left(\psi_{1}, \psi_{2}, \psi_{3}, \psi_{4}\right)\right\}
$$

and the metric

$$
d s^{2}=g_{\mu \nu} d x^{\mu} d x^{\nu}=a^{2}(\eta)\left(d \eta^{2}-d l^{2}\right), \quad a(\eta)=e^{\sigma(\eta)},
$$


where

$$
d l^{2}=\frac{d r^{2}}{1-k r^{2}}+r^{2} d \theta^{2}+r^{2} \sin ^{2} \theta d \phi^{2}
$$

The conformally transformed metric has the form

$$
\bar{g}_{\mu \nu}=\operatorname{diag}\left(1,-\frac{1}{1-k r^{2}},-r^{2},-r^{2} \sin ^{2} \theta\right)
$$

In this section we will restrict our attention to the spatially flat $k=0$ case. Then an exponential solution produces the de Sitter space-time (it is not so for $k \neq 0$ ).

Let us consider the variational derivatives $\delta S_{e q} / \delta \psi_{i}$.

$$
\begin{aligned}
R-\frac{\partial}{\partial \psi_{1}} V\left(\psi_{1}, \psi_{2}, \psi_{3}, \psi_{4}\right) & =0 \\
\square R-\frac{\partial}{\partial \psi_{2}} V\left(\psi_{1}, \psi_{2}, \psi_{3}, \psi_{4}\right) & =0 \\
C^{2}-\frac{\partial}{\partial \psi_{3}} V\left(\psi_{1}, \psi_{2}, \psi_{3}, \psi_{4}\right) & =0, \\
\left(E-\frac{2}{3} \square R\right)-\frac{\partial}{\partial \psi_{4}} V\left(\psi_{1}, \psi_{2}, \psi_{3}, \psi_{4}\right) & =0 .
\end{aligned}
$$

Furthermore, we need the equation for the metric, which can be obtained by taking the derivative of the action with respect to $\sigma, \delta S_{e q} / \delta \sigma=0$. This gives

$$
\begin{aligned}
& -3 e^{-4 \sigma}\left(4 \sigma^{\prime 3} \psi_{2}^{\prime}-2 \psi_{2}^{\prime \prime} \sigma^{2}-4 \psi_{2}^{\prime} \sigma^{\prime} \sigma^{\prime \prime}+\psi_{2}^{\prime \prime \prime \prime}+4 \psi_{2}^{\prime \prime} \sigma^{\prime \prime}+2 \psi_{2}^{\prime} \sigma^{\prime \prime \prime}\right) \\
& -3 e^{-2 \sigma}\left(\psi_{1}^{\prime \prime}+2 \sigma^{\prime \prime} \psi_{1}+2 \psi_{1}^{\prime} \sigma^{\prime}+2 \sigma^{\prime 2} \psi_{1}\right)+2 e^{-4 \sigma} \psi_{4}^{\prime \prime \prime \prime}-2 V\left(\psi_{1}, \psi_{2}, \psi_{3}, \psi_{4}\right)=0 .
\end{aligned}
$$

If we assume that there exists an exponential (in terms of physical time) solution $a=a_{0} e^{H_{0} t}$, it can be inserted into the equations (80) with the following output:

$$
\frac{\partial V}{\partial \psi_{1}}=-12 H_{0}^{2}, \quad \frac{\partial V}{\partial \psi_{2}}=0, \quad \frac{\partial V}{\partial \psi_{3}}=0 \quad \text { and } \quad \frac{\partial V}{\partial \psi_{4}}=24 H_{0}^{4} .
$$

Furthermore, the equation for the conformal factor has the form

$$
\begin{aligned}
& -4 V-24 H_{0}^{2} \psi_{1}-18 H_{0} \dot{\psi_{1}}-6 \ddot{\psi}_{1}-72 \dot{\psi_{2}} H_{0}^{3}-78 H_{0}^{2} \ddot{\psi}_{2} \\
& +36 H_{0} \dot{\dot{\psi}_{2}}+6 \ddot{\psi_{2}}+24 H_{0}^{3} \dot{\psi}_{4}+44 H_{0}^{2} \ddot{\psi}_{4}+24 H_{0} \dot{\dot{\psi}_{4}}=0
\end{aligned}
$$

Finding of dS-like solutions in this way implies resolving the system of equations (81), (82), but it is not clear whether this can be done in a general form. It should be noted that the r.h.s. of the equations (81) - (82) are valid only assuming that we have already used some (unknown) solutions for the auxiliary scalars $\psi_{k}$ there. 
Let us try another approach for de Sitter solutions. Consider first, as a heat-up exercise, the theories with the actions (11) and (2). We know from the Sect. 2 that (1) and (2) are equivalent, provided that the functions $\psi=f_{R}^{\prime}(R)$ and $R=V_{\psi}^{\prime}(\psi)$ are inverse functions. Let us use this fact to obtain the criterion of dS-like solution for the theories (11) and (2). The dS solution means, in the new frame, that

$$
R_{\mu \nu}=\frac{1}{4} R g_{\mu \nu} \quad \text { and } \quad R_{\mu \nu \alpha \beta}=\frac{1}{12} R\left(g_{\mu \alpha} g_{\nu \beta}-g_{\nu \alpha} g_{\mu \beta}\right), \quad \text { also } \quad R=\text { const } .
$$

How can we see whether the solution of the form (83) is possible or not for the given theory? In case of (1) one can take variation with respect to the metric, $\delta g_{\mu \nu}=h_{\mu \nu}$, and arrive at the equation

$$
\frac{1}{2} f g_{\mu \nu}-R_{\mu \nu} f_{R}^{\prime}+\left(\nabla_{\mu} \nabla_{\nu}-g_{\mu \nu} \square\right) f_{R}^{\prime}=0
$$

Inserting (83) into the last equation, we arrive at the well known algebraic equation which roots $R=$ const give us dS solutions of $f(R)$ gravity:

$$
\frac{1}{2} g_{\mu \nu} f-\frac{1}{4} R g_{\mu \nu} f_{R}^{\prime}=0 \quad \Longrightarrow \quad R f_{R}^{\prime}=2 f \text {. }
$$

Now we can do the same for the (2) version of the same theory. The equations equivalent to (84) in this case have the form

$$
\frac{1}{2} g_{\mu \nu}(\psi R-V)-\psi R_{\mu \nu}=0, \quad \text { also } \quad R=V_{\psi}^{\prime} .
$$

Inserting (83) into (86), we arrive at the algebraic equation for constant values of $\psi$ at all possible dS solutions:

$$
\psi V_{\psi}^{\prime}=2 V
$$

It is fairly easy to see that Eq. (87) is nothing else but the mapping of the final relation in Eq. (85). For this end one has to just use our main relations $\psi=f_{R}^{\prime}(R)$ and $R=V_{\psi}^{\prime}(\psi)$ in (85).

One can note that the relation (87) plays exactly the same role for the theory (2) as the relation (85) does play for the theory (1).

The next task is to obtain similar relations for the theories (28) and (29). One can immediately notice that under the conditions (86) we have $X_{1}=\square R=0$ and $X_{3}=C^{2}=0$. Therefore the existence of the solution of the form (86) concerns only the dependence of $f\left(X_{i}\right)$ on $X_{2}$ and $X_{4}$ in one case and the dependence of $V\left(\psi_{i}\right)$ on $\psi_{2}$ and $\psi_{4}$ in another case. In all cases we can also consider $E$ instead of $\tilde{E}$. 
First we deal with Eq. (28). In taking variations of the metric we have to remember that after that we shall integrate by parts and then use the conditions (86). Therefore, all covariant derivatives, either acting on the variation of the metric $h_{\mu \nu}$ or on curvature tensor components, can be safely neglected. In this way we obtain

$$
\delta S_{1}=\int d^{4} x \sqrt{-g}\left\{\frac{1}{2} h f+f_{R}^{\prime} \cdot \delta_{h} R+f_{E}^{\prime} \cdot \delta_{h} E\right\}
$$

A very simple calculations give

$$
\left.\delta_{h} R\right|_{d S}=-\frac{1}{4} R h, \quad \text { and }\left.\quad \delta_{h} E\right|_{d S}=-\frac{1}{12} R^{2} h=-\frac{1}{2} E h
$$

where we denoted $h=h_{\mu}^{\mu}$. Then, for all dS solutions of this theory, the constant invariants $R$ and $E$, related by the consistency condition $E=R^{2} / 4$ in this case, should also satisfy the "on-shell" algebraic equation

$$
f=\frac{1}{2} R f_{R}^{\prime}+E f_{E}^{\prime}
$$

The last equation is a direct generalization of (85) and has the same theoretical status for the more general theory (28) as (85) has for the theory (11). It follows from expressions presented in e.g. [14, 20] (though we have been unable to find a paper where it was written explicitly).

The next step is to obtain the extension of Eq. (87) for the more general case of the theory (29). We take all three necessary variations and get

$$
\begin{aligned}
\frac{\delta S_{2}}{\delta \psi_{1}}=0 \quad \Longrightarrow \quad R=V_{1}^{\prime}=\frac{\partial V}{\partial \psi_{1}} \\
\frac{\delta S_{2}}{\delta \psi_{4}}=0 \quad \Longrightarrow \quad E=V_{4}^{\prime}=\frac{\partial V}{\partial \psi_{4}} \\
\left.\frac{\delta S_{2}}{\delta h_{\mu \nu}}\right|_{d S}=0 \quad \Longrightarrow \quad \frac{1}{2}\left(V-\psi_{2} R-\psi_{4} E\right)=-\frac{1}{4} \psi_{2} R-\frac{1}{2} \psi_{4} E
\end{aligned}
$$

It is easy to see from Eq. (91) that one equation for constant values of $\psi_{i}, i=1,2,3,4$, at the de Sitter solutions (83) has the same form (87) (with $\psi_{1}$ instead of $\psi$ and $V$ depending on all $\psi_{i}$ ) even if the Gauss-Bonnet and other terms are present (of course, this does not mean that these terms play no role here).

The other three algebraic "on-shell" equations follow from (81) by excluding $H_{0}$ (still to be found) and using the relation between $R$ and $E$ for a dS solution:

$$
\frac{\partial V}{\partial \psi_{1}}=0, \quad \frac{\partial V}{\partial \psi_{3}}=0, \quad \frac{\partial V}{\partial \psi_{4}}=\frac{1}{4}\left(\frac{\partial V}{\partial \psi_{2}}\right)^{2} .
$$




\section{STRING-INSPIRED CASE}

Now let us try to generalize the consideration given above to more general gravitational actions containing more complicated terms constructed from the scalar curvature $R$, the Ricci tensor $R_{\mu \nu}$ and the Riemann tensor $R_{\mu \nu \alpha \beta}$. In general, the corresponding theories can not be reduced to the metric-scalar models. For example, the $R_{\mu \nu \alpha \beta}^{2}$-type actions involve higher derivatives not only in the spin-zero sector but also in the spin-two one. Hence one can expect that the reduction to second order equations would require introduction of tensor compensating fields. This is definitely true in general, however there is an interesting possibility yet. Let us consider a special space-time metric with a restricted number of degrees of freedom, such as the cosmological FRW one. In this case we have only one component of the metric - the scale (conformal) factor, which depends on a single variable (e.g. conformal time). Then we meet a much simpler situation than in the general case, because the tensor structure of the $f\left(R_{\mu \nu \alpha \beta}\right)$ action becomes irrelevant. It might happen that the reduction to the metric-scalar theory will be possible in this case. Indeed, this reduction concerns only the dynamics of the conformal factor of the metric. After this dynamics is described in terms of an appropriate metric-scalar theory, one has to explore other, more complicated aspects (e.g. metric perturbations) in the framework of the original higher-derivative theory. However, the equivalence with the metric-scalar model may be a useful tool for dealing with a homogeneous and isotropic cosmological solution. Therefore, it deserves our attention.

Let us consider an effective low energy action of (super)string theory (see, e.g. [21]) depending only on metric. For the sake of simplicity we assume that the dilaton and effective fermionic degrees of freedom (after compactification to $d=4$, because we are interested in the four-dimensional physics here) are all in the vacuum state and only metric possesses dynamical behaviour. This assumption is perfectly consistent with the known form of the gravitational interaction which corresponds to General Relativity (GR), the theory where only massless graviton is propagating.

In the lowest, first order in the string parameter $\alpha^{\prime}$, we meet the standard Einstein-Hilbert action for gravity. In the next order in $\alpha^{\prime}$ we meet a set of higher derivative terms, namely

$$
R_{\mu \nu \alpha \beta}^{2}, \quad R_{\mu \nu}^{2} \quad \text { and } \quad R^{2}
$$

It has been noticed 25 years ago by Zwiebach 22] (see also consequent investigation of the problem in 23] ) that the choice of the background string metric can be always done in such a way that the higher order corrections do not generate unphysical propagating massive ghosts - a typical phenomenon for a wide class of higher derivative gravity theories [24, 25]. In the second order in 
$\alpha^{\prime}$, the ghosts do not show up if the higher derivative terms (93) enter the following combinations

$$
E=R_{\mu \nu \alpha \beta}^{2}-4 R_{\mu \nu}^{2}+R^{2}, \quad R^{2}
$$

Possible metric reparametrizations in the $\mathcal{O}\left(\alpha^{\prime 2}\right)$ order have the form

$$
g_{\mu \nu} \longrightarrow g_{\mu \nu}^{\prime}=g_{\mu \nu}+\alpha^{\prime}\left(x_{1} R_{\mu \nu}+x_{2} R g_{\mu \nu}\right)+\ldots
$$

where $x_{1,2}$ are arbitrary parameters. The same procedure can be used in higher orders. The above transformations can change coefficients (in particular eliminate completely) of all those terms which depend on the Ricci tensor or on the scalar curvature $R$, only those terms which are constructed exclusively from the Riemann tensor may not be modified. The next question is what are the physical constraints for those terms which can be modified. As we have already seen above, the $R^{2}$ term can be traded for a scalar field. The first term in the last expression is nothing else but the integrand of the Gauss-Bonnet topological invariant (Euler characteristics) of the space-time manifold. It is remarkable that this term does not influence the dynamics of the universe. Hence one can completely eliminate the relevant $\mathcal{O}\left(R^{2}\right)$ corrections to the string effective action by means of the metric reparametrization. At the same time, this procedure is not uniquely defined. If we require the absence of unphysical ghosts, we may eliminate or not the relevant $R^{2}$ term depending on our own will. The related ambiguity may affect the cosmological solutions [26] and can not be fixed without experimental verification (see also corresponding discussion for the case of the gravity with dilaton and torsion in [23] and [27]). The $R+R^{2}$-type action corresponds to the choice of the metric parametrization described above.

In the next order in $\alpha^{\prime}$, we meet corrections which are cubic in curvature tensor and also the $R \square R$-type terms. Since the latter term generically leads to the appearance of a ghost scalar [7, 28], we investigate the most general metric parametrization in this given order of the former term only. The corresponding effective action has the form

$$
\begin{aligned}
S_{3}=\int d^{4} \sqrt{-g} & \left\{x_{1} R^{3}+x_{2} R R_{\mu \nu} R^{\mu \nu}+x_{3} R R_{\mu \nu \alpha \beta} R^{\mu \nu \alpha \beta}\right. \\
& \left.+x_{4} R_{\mu \nu} R^{\nu \alpha} R_{\alpha}^{\mu}+x_{5} R_{\mu \nu \alpha \beta} R^{\alpha \beta \rho \lambda} R^{\mu \nu}{ }_{\rho \lambda}\right\} .
\end{aligned}
$$

Let us now consider the possibility of reduction of the above theory (96) to the metric-scalar model in case of the special conformally flat metric $g_{\mu \nu}=\bar{g}_{\mu \nu} a^{2}(\eta)$. Here $\eta$ is the conformal time and $\bar{g}_{\mu \nu}$ is the time-independent homogeneous and isotropic metric (78). For our purposes it is better to use the variable $\sigma(\eta)$, defined in (76). The transformation rules for the curvature scalar 
and tensors has the form

$$
\begin{aligned}
R & =e^{-2 \sigma}\left[\bar{R}-6(\bar{\nabla} \sigma)^{2}-6 \square \sigma\right] \\
R_{\mu \nu} & =\bar{R}_{\mu \nu}-2\left(\bar{\nabla}_{\mu} \bar{\nabla}_{\nu} \sigma\right)-\bar{g}_{\mu \nu}(\square \sigma)+2\left(\bar{\nabla}_{\mu} \sigma\right)\left(\bar{\nabla}_{\nu} \sigma\right)-2 \bar{g}_{\mu \nu}(\bar{\nabla} \sigma)^{2}, \\
R_{\mu \nu \alpha \beta} & =e^{2 \sigma}\left[\bar{R}_{\mu \nu \alpha \beta}+\left(\bar{g}_{\mu \beta} \bar{g}_{\alpha \nu}-\bar{g}_{\mu \alpha} \bar{g}_{\beta \nu}\right)(\bar{\nabla} \sigma)^{2}\right. \\
& +\left(\bar{g}_{\alpha \nu} \bar{\nabla}_{\mu} \bar{\nabla}_{\beta} \sigma-\bar{g}_{\alpha \mu} \bar{\nabla}_{\nu} \bar{\nabla}_{\beta} \sigma+\bar{g}_{\beta \mu} \bar{\nabla}_{\nu} \bar{\nabla}_{\alpha} \sigma-\bar{g}_{\beta \nu} \bar{\nabla}_{\mu} \bar{\nabla}_{\alpha} \sigma\right) \\
& \left.+\left(\bar{g}_{\alpha \mu} \bar{\nabla}_{\nu} \sigma \bar{\nabla}_{\beta} \sigma-\bar{g}_{\alpha \nu} \bar{\nabla}_{\mu} \sigma \bar{\nabla}_{\beta} \sigma-\bar{g}_{\beta \mu} \bar{\nabla}_{\nu} \sigma \bar{\nabla}_{\alpha} \sigma+\bar{g}_{\beta \nu} \bar{\nabla}_{\mu} \sigma \bar{\nabla}_{\alpha} \sigma\right)\right] .
\end{aligned}
$$

For the metric of interest we arrive at the relations for the non-zero components of the above curvatures

$$
\begin{aligned}
R & =e^{-2 \sigma}\left[\bar{R}-6{\sigma^{\prime}}^{2}-6 \sigma^{\prime \prime}\right] \\
R_{\eta \eta} & =-3 \sigma^{\prime \prime}, \quad R_{i j}=\bar{g}_{i j}\left(\frac{1}{3} \bar{R}-\sigma^{\prime \prime}-2 \sigma^{\prime 2}\right) \\
R_{\eta i \eta k} & =-e^{2 \sigma} \sigma^{\prime \prime} \bar{g}_{i j}, \quad R_{i j k l}=e^{2 \sigma}\left(\frac{1}{6} \bar{R}-{\sigma^{\prime}}^{2}\right)\left(\bar{g}_{i k} \bar{g}_{j l}-\bar{g}_{i l} \bar{g}_{j k}\right),
\end{aligned}
$$

where $\bar{R}=-6 k=$ const.

Using the relations (100) - (102), after some algebra we can rewrite the elements of the action (96) in the form

$$
\begin{aligned}
& \sqrt{-g} R^{3}=e^{2 \sigma}\left[\bar{R}-6{\sigma^{\prime}}^{2}-6 \sigma^{\prime \prime}\right]^{3}
\end{aligned}
$$

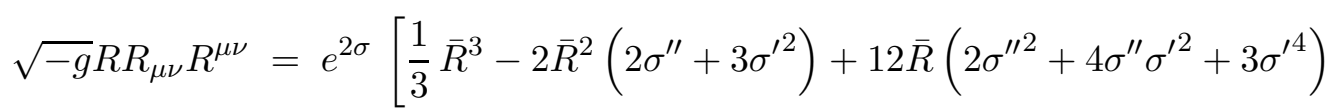

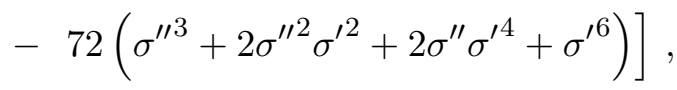

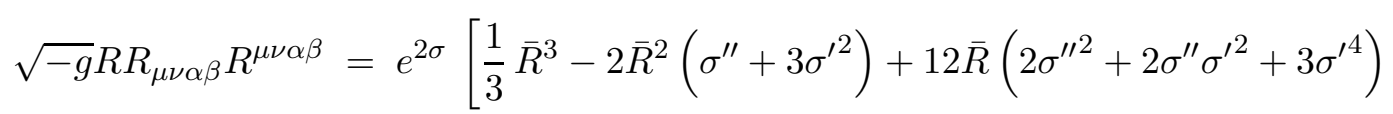

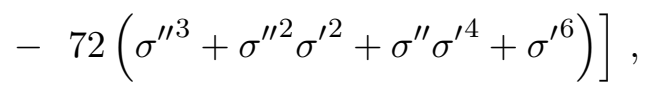

$$
\begin{aligned}
& \sqrt{-g} R_{\mu \nu} R^{\nu \alpha} R_{\alpha}^{\mu}=e^{2 \sigma}\left[\frac{1}{9} \bar{R}^{3}-\bar{R}^{2}\left(\sigma^{\prime \prime}+2{\sigma^{\prime}}^{2}\right)+3 \bar{R}\left(\sigma^{\prime \prime}+2{\sigma^{\prime}}^{2}\right)^{2}\right. \\
& -6\left(5{\sigma^{\prime \prime}}^{3}+3{\sigma^{\prime \prime 2}}^{\prime 2}+6{\sigma^{\prime \prime}}^{\left.\left.\sigma^{4}+4{\sigma^{\prime}}^{6}\right)\right]}\right. \text {, } \\
& \sqrt{-g} R_{\mu \nu \alpha \beta} R_{\rho \beta \rho \lambda}^{\mu \nu} R_{\rho \lambda}=e^{2 \sigma}\left[\frac{1}{9} \bar{R}^{3}-2 \bar{R}^{2}{\sigma^{\prime 2}}^{2}+12 \bar{R}{\sigma^{\prime}}^{4}-24\left({\sigma^{\prime \prime 3}}^{3}+{\sigma^{\prime}}^{6}\right)\right]
\end{aligned}
$$


The sufficient condition of the reduction to a metric-scalar theory can be easily found by using analogy with the $\sqrt{-g} R^{3}$ case considered in the previous sections. In the general case this condition has the form

$$
\sqrt{-g}\left(x_{1} R^{3}+x_{2} R R_{\mu \nu}^{2}+x_{3} R R_{\mu \nu \alpha \beta}^{2}+x_{4} R_{\mu \nu}^{3}+x_{5} R_{\mu \nu \alpha \beta}^{3}\right)=e^{2 \sigma}\left[y_{1} \bar{R}^{3}+y_{2} \sigma^{\prime \prime}+y_{3}{\sigma^{\prime}}^{2}\right]^{3},
$$

where $y_{1,2,3}$ are some additional arbitrary coefficients. Thus we obtain 10 algebraic equations for the 8 variables $x_{1,2,3,4,5}$ and $y_{1,2,3}$. In fact, the number of the variables can be immediately reduced to 7 by noticing that the coefficient $x_{5}$ can not be made zero in the string induced gravity by means of the metric reparametrization, while all other coefficients can [22]. Therefore without losing generality we can set $x_{5}=1$. Now, as far as the number of equations is much greater than the number of independent variables, it is not certain that the solution of these equations exist. As we shall immediately see, it exist only for some particular, but the most relevant case. Let us remember that our prime interest is the inflationary epoch, where the space curvature is negligible. Then, as a first step we can look for the solution in the simplest $k=0$ case, where $\bar{R}=0$ and the number of equations is even smaller than the number of independent variables. In this case the equations become

$$
\begin{aligned}
-y_{2}^{3} & =216 x_{1}+72 x_{2}+72 x_{3}+30 x_{4}+24, \\
-3 y_{2}^{2} y_{3} & =648 x_{1}+144 x_{2}+72 x_{3}+18 x_{4}, \\
-3 y_{2} y_{3}^{2} & =648 x_{1}+144 x_{2}+72 x_{3}+36 x_{4}, \\
-y_{3}^{3} & =216 x_{1}+72 x_{2}+72 x_{3}+24 x_{4}-24 .
\end{aligned}
$$

Using the pairs of equations (110), (111) and (109), (112) we obtain the relation

$$
y_{3} y_{2}\left(y_{3}-y_{2}\right)=\left(y_{3}-y_{2}\right)\left(y_{3}^{2}+y_{3} y_{2}+y_{2}^{2}\right)=6 x_{4} \text {. }
$$

These equations can be satisfied only for $y_{2}=y_{3}=y$ and $x_{4}=0$. Let us remark that the constraint $y_{1}=y_{2}$ means that possible dependence on the conformal factor for the theories reducible to metricscalar models can be only the same as for the $\sqrt{-g} R^{3}$-case. All other choices are not reducible. The general solution corresponds to

$$
x_{1}=-\frac{x}{3}-\frac{2}{9}+\frac{y^{3}}{216}, \quad x_{2}=-2 x-1+\frac{y^{3}}{36}, \quad x_{3}=x, \quad x_{5}=1,
$$

where $x, y$ are arbitrary parameters. It is easy to see that if the constrains (114) are satisfied, we have the same reduction to a metric-scalar model as in the case of the higher derivative term

$$
S=-\frac{y^{3}}{216} \int d^{4} x \sqrt{-g} R^{3}
$$


At the level of the metric-scalar model we have, in the string-induced case, exactly the same situation as in the case of the term (115).

For the sake of completeness, we consider the more complicated $k \neq 0$ case. An important observation is that the constraints (114) must hold also for $k \neq 0$. Then elementary analysis shows that in this case there are no solutions of eq. (108). Therefore, mathematically the reduction to the metric-scalar model can not be exact in the general case, but only an approximate one. However the quality of this approximation is indeed excellent because the role of $k$ during inflation is negligible.

\section{METRIC DUAL FOR THE EDDINGTON-LIKE GRAVITY}

As another illustration of the effectiveness of our approach, consider the Eddington-like action of gravity [29, 30] [48],

$$
S_{\mathrm{Edd}}=\alpha \int \sqrt{\left|R_{\mu \nu}\right|} d^{4} x
$$

Here and in what follows we use the notation $\left|R_{\mu \nu}\right|=\left|\operatorname{det}\left(R_{\mu \nu}\right)\right|$, furthermore $\alpha$ is a dimensionless parameter and $R_{\mu \nu}$ is the (symmetric) Ricci tensor constructed from the symmetric affine connection $\Gamma_{\mu \nu}^{\lambda}$. The action (116) is equivalent to the vacuum Einstein-Hilbert action with a non-zero cosmological constant [31, 33]. In what follows we will show it is straightforward to achieve the same result by using the method we have considered in the previous sections. Moreover, in the known approaches [33, 34], it is common to consider the first order formalism for gravity, taking $\Gamma_{\mu \nu}^{\lambda}$ to be independent from the metric $g_{\mu \nu}$. Our method does not require this restriction and, moreover, $R_{\mu \nu}$ can be traded for any other symmetric tensor, e.g. to some combination of the torsion fields (e.g., the one considered in [27]). Of course, the corresponding model will not be equivalent to the GR, but it can be mapped to a dual theory in the same way as we will describe below.

The dual equivalent action should have the form

$$
S_{e q}=\int d^{4} x\left\{J^{\mu \nu} \cdot R_{\mu \nu}-V\left(J^{\mu \nu}\right)\right\},
$$

where $J^{\mu \nu}$ is an auxiliary field. One can immediately note that there is a unique functional form of the potential function $V(J)=V\left(J^{\mu \nu}\right)$ which is compatible with the covariance of the action (117). Indeed, the covariance requires that $J^{\mu \nu}$ should be a tensor density and there must be such symmetric tensor quantity $\Phi_{\mu \nu}$, such that

$$
J^{\mu \nu}=\sqrt{\Phi} \Phi^{\mu \nu}
$$


where $\Phi^{\mu \alpha} \cdot \Phi_{\alpha \nu}=\delta_{\nu}^{\mu}$ and $\Phi=\left|\operatorname{det}\left(\Phi_{\mu \nu}\right)\right|$. This relation (118) can be easily inverted, so we get

$$
\Phi=\operatorname{det}\left(\Phi_{\mu \nu}\right)=\frac{1}{\operatorname{det}\left(J^{\mu \nu}\right)} \quad \text { and } \quad \Phi^{\mu \nu}=\frac{1}{\sqrt{\left|\operatorname{det}\left(J^{\mu \nu}\right)\right|}} J^{\mu \nu}
$$

As a result, we arrive at $V\left(J^{\mu \nu}\right)=k \cdot \sqrt{\Phi}$, where $k$ is some constant. It is easy to see from the relations (119) that this means

$$
V\left(J^{\mu \nu}\right)=\frac{k}{\sqrt{\mid \operatorname{det}\left(J^{\mu \nu} \mid\right.}} .
$$

Now let us see whether we can arrive at the same result (117), (120) by using the method described in the previous sections. By taking derivative of the function $f\left(R_{\mu \nu}\right)=\alpha \sqrt{\left|R_{\mu \nu}\right|}$ and inverting it, we obtain

$$
J^{\mu \nu}=\frac{\partial f}{\partial R_{\mu \nu}} \quad \text { and } \quad R_{\mu \nu}=\frac{\partial V}{\partial J^{\mu \nu}} .
$$

The equivalence of the two formulations requires that $J^{\mu \nu}=J^{\mu \nu}\left(R_{\alpha \beta}\right), \quad R_{\mu \nu}=R_{\mu \nu}\left(J^{\alpha \beta}\right)$ and, also, that

$$
J^{\mu \nu} \cdot R_{\mu \nu}-V\left(J^{\mu \nu}\right)=\alpha \sqrt{\left|R_{\mu \nu}\right|}
$$

Now, taking the partial derivatives $\partial / \partial J^{\mu \nu}$ of the equality (122), after some simple algebra we arrive at

$$
J^{\mu \nu}=\frac{\alpha}{2} \sqrt{\left|R_{\mu \nu}\right|}\left(R_{. .}^{-1}\right)^{\mu \nu}
$$

where $\left(R_{. .}^{-1}\right)^{\mu \nu}$ means the matrix inverse to $R_{\mu \nu}$. It is easy to obtain the relation

$$
\left(R_{. .}^{-1}\right)^{\mu \nu}=\frac{\alpha}{2} \frac{J^{\mu \nu}}{\sqrt{\mid \operatorname{det}\left(J^{\mu \nu} \mid\right)}},
$$

which shows that $J^{\mu \nu}$ is a tensor density. The corresponding tensor field is defined through the relation (118). After some small algebra we arrive at the equation

$$
R_{\mu \nu}=\frac{2}{\alpha} \Phi_{\mu \nu}=\frac{\partial V}{\partial J^{\mu \nu}}
$$

Finally, integrating this equation we get the expected result

$$
V\left(J^{\mu \nu}\right)=\frac{4}{\alpha \sqrt{\left|\operatorname{det}\left(J^{\mu \nu}\right)\right|}}=\frac{4}{\alpha} \sqrt{\Phi},
$$

that is nothing else but Eq. (120) with $k=4 / \alpha$.

It is clear that the consideration presented above opens the way for some interesting applications. One can, at the first place, identify the auxiliary tensor quantity $\Phi_{\mu \nu}$ directly with the space-time 
metric $g_{\mu \nu}$, but this is not the only one possible choice. Let us note that the real identification of the metric occurs when matter is introduced into the theory. One can assume, for instance, that there is a scaling relation $\Phi_{\mu \nu}=\lambda \cdot g_{\mu \nu}$ or even more complicated one, like $\Phi_{\mu \nu}=B(x) \cdot g_{\mu \nu}$, where the scalar field $B(x)$ depends on the space-time coordinates. The corresponding generalization of the Eddington-like gravity theory looks interesting and perhaps deserves further discussion. The method presented here can be immediately extended to the case when $R_{\mu \nu}$ in the initial action (116) is traded for some other tensor, e.g. for $R_{\mu \nu}+C g_{\mu \nu}$. In this case the auxiliary field $\Phi_{\mu \nu}$ is naturally identified with the second metric and we arrive at the bimetric theory of gravity [31].

\section{A BRIEF NOTE ON QUANTUM (NON)EQUIVALENCE}

In the previous sections $2-4$ and 5 we have presented several examples of classically equivalent theories. It looks interesting to see what happens with this equivalence at the quantum level. It is well known that the quantum equivalence does not imply the quantum one. One can find statements of this type in Refs. [9, 11] and also in [17]. Of course, classical equivalence leads to quantum equivalence at the level of tree diagrams or imaginary parts of one-loop diagrams which, in particular, describe creation of real particles and field perturbations by external gravitational fields and do not need renormalization. That is why primordial spectra of scalar and tensor perturbations generated during inflation in $f(R)$ gravity (11) (in the models which admit it, say, in the $R+R^{2}$ model [35]) coincide with those for inflationary models with a non-minimally coupled scalar field in the limit of a large negative coupling $|\xi| \gg 1$, see e.g. [36 38], since the latter models reduce to the form (2) after neglecting the kinetic term of the scalar field that is justified in this limit. Note that the Higgs inflation [39] belongs to this class, too.

The problems of establishing general quantum equivalence become much more complicated when taking into account the need for renormalization. For the sake of simplicity we consider the theories (11) and (2), but the generalizations to other cases are indeed possible.

The quantum equivalence at the one-loop level means, at the first place, that the relations like $\psi=f^{\prime}(R)$ and $R=V^{\prime}(\psi)$ do hold for the one-loop counterterms. In reality, this is not necessary so, because these relation may also require renormalization. Moreover, even if the oneloop divergences do satisfy this requirement, it is very unlikely that some sort of relation between the two (classically equivalent) theories will hold beyond one-loop approximation.

The main difficulty of discussing the equivalence of the two formulations, e.g., (11) and (2), or (27) and (28) - is the fact that the corresponding theories are not renormalizable. At the same time, 
even one-loop divergences may be non-equivalent, as it happens with the tensor-scalar model in different conformal frames [40,41]. Indeed, the non-equivalence here means that one can not easily find an explicit transformation which would link the two expressions for one-loop counterterms in the two representations. At the same time, since both theories are non-renormalizable, one can speak about certain qualitative equivalence in a sense that, in both frames, the necessary counterterms have the structure distinct from the one of the initial action.

One can look from another side and compare the UV completion of the two, classically equivalent, theories. It is easier to perform such discussion for a more general cases of the theories (27) and (28). The theory (28) with "frozen" scalars $\psi^{i}$ is renormalizable [25] (see also [42] for a more detailed introduction). Therefore, in order to construct renormalizable theory out of (28), one has to complete the action by certain second- and fourth-derivative terms constructed from the scalars $\psi^{i}$. In case of one scalar field this completion has been considered in [43] and more recently in [44] in relation to inflation. The generalization to the many-scalar case is obvious, but the output would be quite cumbersome. The situation with the general higher-derivative model (27) is much more complicated. First of all, there is a very strong difference between polynomial and non-polynomial functions $f\left(X_{i}\right)$. In the last case the problem of quantum formulation is unclear (despite potentially interesting, see e.g., [45]). Contrary to that, in the polynomial case the prescription for constructing renormalizable and super-renormalizable theories of quantum gravity is known [24]. If the highest power of curvature tensor in $f\left(X_{i}\right)$ is $N \geq 3$, one has to introduce into Lagrangian all possible covariant term of this dimension, including the additional terms of the following form:

$$
\sum_{k=0}^{N-2}\left(\alpha_{k} R_{\mu \nu} \square^{k} R^{\mu \nu}+\beta_{k} R \square^{k} R\right) .
$$

If the largest order coefficients $\alpha_{N-2}$ and $\beta_{N-2}$ are nonzero, the theory is super-renormalizable [49].

Now we are in a position to compare the renormalizability properties of the theories (27) and (28). The UV completions described above correspond to the possible counterterms and, in these two cases, these counterterms are dramatically different. For example, the UV completion in the case of scalar-tensor theory (28) describes the propagation of only two spin-2 states, namely of graviton and of the massive spin-2 ghost. At the same time the UV completion of the theory (27) has (for $N \geq 3$ ) at least one more spin-2 massive particle [24]. Thus, we can conclude that the renormalization properties of the two classically equivalent non-renormalizable theories are, in general, quite different. In particular, they have very distinct UV completions and, consequently, 
very distinct structure of couterterms.

\section{CONCLUSION}

We have described in the previous sections how to perform a mapping of a gravitational theory of the type $f\left(X^{i}\right)$ into a theory with auxiliary scalar fields. The number of these fields is determined by the rank of the Hessian matrix of $f\left(X^{i}\right)$. The scheme which we have described is more general than the ones known before. In particular, it enables one to deal with the constrained case and leads to an auxiliary-field representations for the actions which look like renormalization-group corrected vacuum actions in gravity theories like Eq. (49). On the top of this we have formulated the general conditions for the exponential expansion of the Universe and extended the analysis for the string-inspired case, where the treatment with auxiliary scalars is possible only for a FRW-like solution. Finally, we have shown that our approach is perfectly applicable to the Eddington-like gravity models and to a wide class of their extensions. Depending on the initial model, our approach shows either how the metric emerges in a theory which had, initially, only affine connection, or leads to a bimetric theories of gravity.

\section{Acknowledgments}

D.R. thanks the Departamento de Matemática Aplicada at Unicamp, SP, Brazil, where part of this work was done, M. Bañados and A. Gomberoff for useful comments on the Eddington action and FAPESP for partial financial support. F.S. and I.Sh. are grateful to FAPEMIG and CNPq for partial support. I.Sh. was also supported by ICTP visiting program. A.S. acknowledges the RESCEU hospitality as a visiting professor. He was also partially supported by the Russian Foundation for Basic Research under grant 09-02-12417-ofi-m.

[1] T. P. Sotiriou and V. Faraoni, Rev. Mod. Phys. 82 (2010) 451 arXiv:0805.1726;

A. De Felice and S. Tsujikawa, Living Rev. Rel. 13 (2010) 3 arXiv:1002.4928.

[2] J. O'Hanlon, Phys. Rev. Lett. 29, 137 (1972).

[3] P. Teyssander and P. Tourrenc, J. Math. Phys. 24, 2793 (1983).

[4] T. Chiba, Phys. Lett. B 575, 1 (2003) arXiv:astro-ph/0307338.

[5] B. Whitt, Phys. Lett. B 145 (1984) 176;

K.-i. Maeda, Phys. Rev. D 39 (1989) 3159;

J. D. Barrow and S. Cotsakis, Phys. Lett. B 258 (1991) 299;

G. Magnano and L.M. Sokolowski, Phys. Rev. D 50 (1994) 5039. 
[6] D. Wands, Class. Quantum Grav. 11 (1994) 269.

[7] S. Gottlober, H. J. Schmidt and A. A. Starobinsky, Class. Quantum Grav. 7 (1990) 893.

[8] Sh. Nojiri and S. D. Odintsov, Gen. Rel. Grav. 36 (2004) 1765 arXiv:hep-th/0308176;

G. Cognola, E. Elizalde, Sh. Nojiri, S. D. Odintsov and S. Zerbini, JCAP 0502 (2005) 010 arXiv:hep-th/0501096;

Sh. Nojiri and S. D. Odintsov, Lectures at 42nd Karpacz Winter School of Theoretical Physics, Int. J. Geom. Meth. Mod. Phys. 4 (2007) 115 arXiv:hep-th/0601213.

[9] N. Deruelle, Y. Sendouda and A. Youssef, Phys. Rev. D80 (2009) 084032 arXiv:0906.4983.

[10] N. Deruelle, M. Sasaki, Y. Sendouda and D. Yamauchi, Prog. Theor. Phys. 123 (2010) 169 arXiv:0908.0679.

[11] H.- J. Schmidt, Phys. Rev. D49 (1994) 6354; Erratum-ibid. D54 (1996) 7906 arXiv:gr-qc/9404038.

[12] V. Faraoni, E. Gunzig and P. Nardone, Fund. Cosmic Phys. 20 (1999) 121 arXiv:gr-qc/9811047].

[13] D. I. Kaiser, Phys. Rev. D 81 (2010) 084044 arXiv:1003.1159.

[14] A. De Felice and T. Suyama, JCAP 0906 (2009) 034 arXiv:0904.2092.

[15] A. De Felice and T. Tanaka, Progr. Theor. Phys. 124 (2010) 503 arXiv:1006.4399.

[16] I. L. Shapiro, Class. Quantum Grav. (Topical review) 25 (2008) 103001 arXiv:0801.0216.

[17] I. D. Saltas and M. Hindmarsh, The dynamical equivalence of modified gravity revisited, arXiv:1002.1710.

[18] D. M. Gitman and I. V. Tyutin, Quantization of Fields with Constraints (Springer Series in Nuclear and Particle Physics, 1990).

[19] M. Henneaux and C. Teitelboim, Quantization of gauge systems (Princeton, 1992).

[20] S. M. Carroll et. al., Phys. Rev. D 71 (2005) 063513 arXiv:astro-ph/0410031.

[21] M. B. Green, J. B. Schwarz and E. Witten, Superstring Theory (Cambridge University Press, Cambridge, 1987).

[22] B. Zwiebach, Phys. Lett. B 156 (1985) 315.

[23] S. Deser and A. N. Redlich, Phys. Lett. B 176 (1986) 350;

B. E. Fridling and A. Jevicki, Phys. Lett. B 174 (1986) 75;

D. R. T. Jones and A. M. Lowrence, Z. Phys. C 42 (1989) 153;

A. A. Tseytlin, Phys. Lett. B 176 (1986) 92.

[24] M. Asorey, J. L. López and I. L. Shapiro, Int. Journ. Mod. Phys. A 12 (1997) 5711.

[25] K. S. Stelle, Gen. Rel. Grav. 9 (1978) 353.

[26] A. L. Maroto and I. L. Shapiro, Phys. Lett. B 414 (1997) 34 arXiv:hep-th/9706179.

[27] I. L. Shapiro, Phys. Repts. 357 (2002) 113 arXiv:hep-th/0103093.

[28] A. Hindawi, B. A. Ovrut and D. Waldram, Phys. Rev. D 53 (1996) 5597 arXiv:hep-th/9509147.

[29] A. S. Eddington, The mathematical theory of Relativity (Cambridge University Press, Cambridge 1924).

[30] E. Schroedinger, Space-time structure (Cambridge University Press, Cambridge 1950).

[31] M. Banados, A. Gomberoff, D. C. Rodrigues and C. Skordis, Phys. Rev. D 79 (2009) 063515 arXiv:0811.1270.

[32] M. Banados and P. G. Ferreira, Eddington's theory of gravity and its progeny, arXiv:1006.1769

[33] M. Ferraris and J. Kijowski, Gen. Rel. Grav. 14 (1982) 165.

[34] E. S. Fradkin and A. A. Tseytlin, Ann. Phys. 162 (1985) 31.

[35] A. A. Starobinsky, Phys. Lett. 91 (1980) 99.

[36] T. Chiba and M. Yamaguchi, JCAP 0810 (2008) 021 arXiv:0807.4965.

[37] A. O. Barvinsky, A. Yu. Kamenshchik and A. A. Starobinsky, JCAP 0811 (2008) 021 arXiv:0809.2104.

[38] A. Linde, M. Noorbala and A. Westfal, JCAP 1103 (2011) 013 arXiv:1101.2652.

[39] F. L. Bezrukov and M. Shaposhnikov, Phys. Lett. B 659 (2008) 703 arXiv:0710.3755.

[40] I. L. Shapiro and H. Takata, Phys. Rev. D52 (1995) 2162 arXiv:hep-th/9502111.

[41] C. F. Steinwachs and A. Yu. Kamenshchik, One-loop divergences for gravity non-minimally coupled to a multiplet of scalar fields: calculation in the Jordan frame. I. The main results, arXiv:1101.5047,

[42] I. L. Buchbinder, S. D. Odintsov and I. L. Shapiro, Effective Action in Quantum Gravity (IOP Publishing, Bristol, 1992).

[43] E. Elizalde, A. G. Zheksenaev, S. D. Odintsov and I. L. Shapiro, Phys. Lett. B328 (1994) 297 arXiv:hep-th/9402154;

E. Elizalde, S. D. Odintsov and I. L. Shapiro, Class. Quant. Grav. 11 (1994) 1607 arXiv:hep-th/9404064.

[44] S. Weinberg, Phys. Rev. D77 (2008) 123541 arXiv:0804.4291.

[45] E. T. Tomboulis, Super-renormalizable gauge and gravitational theories, arXiv:hep-th/9702146

[46] P. Horava, Phys. Rev. D79 (2009) 084008 arXiv:0901.3775.

[47] Recently, this similarity has been noted in [17].

[48] Recently, the Eddington-like action (116) has became an object of a stronger interest (see, e.g. 31, 32] and references therein). 
[49] The renormalizability by power counting in this theory is exactly the same as in the Horava-Lifshits gravity [46]. 